\title{
Site selection of LNG terminal based on cloud matter element model and principal component analysis
}

\author{
Gaolei Liu*, Ran Dai, Feng Zhang, Yanpeng Zhao, Chaoyue Zhang, and Fucheng Huang \\ Navigation College, Dalian Maritime University, Liaoning, China
}

\begin{abstract}
With the development of liquefied natural gas(LNG) port, as one of the crucial LNG port sitting process, the LNG terminal site's condition assessment method has always received attention from experts, scholars concern more and more about the method's practicality and reliability. In the traditional condition assessment method, due to the characteristics of the complex and extensive factors in the comprehensive assessment of the LNG terminal site, the assessment system is not comprehensive enough, or the assessment is too complex, the indexes are not easy to quantify, such problems are emerging. In view of the above reasons, the principal component analysis(PCA) method is used to transform the multi-indicators that affect the comparison of terminal sites into a few comprehensive indicators. A comprehensive evaluation model of the LNG terminal site based on cloud matter element theory and subjective and objective comprehensive weighting method was constructed. By the subjective and objective comprehensive weighting method, the comprehensive weight of each index is determined and the LNG terminal site comprehensive assessment standard cloud element model is constructed with the combination of cloud model and matter-element theory. The cloud matter-element correlation function is established to determine the degree of association between the matter element to be evaluated and the standard cloud matter element model. In order to eliminate random errors and improve the credibility of the results, the algorithm is used for multiple calculations and analysis to achieve the purpose of simultaneously giving the evaluation results and coefficients of credible degree. Finally, the reliability and rationality of the method are verified by an example.
\end{abstract}

\section{Introduction}

With the increasing proportion of global energy consumption and supply of Liquefied Nature Gas(LNG), major ports along the world have actively responded to and constructed LNG receiving stations and pipeline layout projects. In the actual site selection process, there are often multiple terminal location options in the same LNG project area. In order to avoid blind selection, and provide a certain scientific basis for the terminal site comparison.

\footnotetext{
* Corresponding author: golea520@163.com
} 
This paper carried out research on the comparison and selection of LNG terminal sites. The comparison and selection of LNG terminal site refers to an important task of selecting multiple candidate sites, which plays a role in escorting the construction and operation of the receiving station.

Due to the complex factors of the comparison, evaluation and selection of LNG terminal sites, for a long time, the means most widely used have been the comprehensive evaluation method of experts. By selecting the construction sites based on the individual experience, knowledge and subjective judgment of experts, this often lead to the results of authoritative experts decisively. Because the period of LNG industry development is not so long, there is not much literature and paper on the selection of LNG terminal sites. Although the industry has applied a large number of comprehensive evaluation mathematical models, such as the linear weighted evaluation method [1], the nonlinear weighted evaluation method, and the technique order preference ideal solution method (TOPSIS), There is the lack of a sound theoretical system and technical support which subjectively influenced by human factors. Hao proposed a fuzzy comprehensive evaluation model for LNG terminal port based on fuzzy mathematics theory [2], which opened up a new way for terminal port site comparison and selection research, but fuzzy mathematics theory relied too much on human's evaluation to make the model applicable and feasible. At the same time, the limitations of the judgment results are increased.

Based on the existing research results, this paper takes into account the inadequacies of previous research, and uses principal component analysis to transform multiple-indicators that affect comparisons and selections of receiving stations into a few comprehensive indicators. Through the principle of additive integration, the subjective weight of the LNG terminal assessment index are determined by the analytic hierarchy process(AHP) and the objective weight of each indicator are calculated by the variation coefficient method (VCM), and the comprehensive weight of each indicator is integrated. A comprehensive assessment method based on cloud-matter element model and principal component analysis is proposed by integrated with cloud model and matter-element theory which can reflect the double uncertainty of evaluation and qualitative and quantitative analysis and processing problems.

\section{Establishment and optimization of the LNG terminal site assessment system}

At the first step of the site selection of LNG terminal, a perfect condition assessment system need to be built at the beginning. Each factor of LNG terminal site assessment may lead a big loss, so we need to set up a complete system. But a very complete system may be too complicated to do the assessment work. There need an appropriate and necessary method to simplify the system. Principal component analysis(PCA) is a good choice to deal with this problem [3] [4].

\subsection{The factors to consider of LNG terminal site assessment system}

Refer to the specification of site selection and design for LNG ports and jetties [5] [6] [7] [8], the factors to consider of site selection for LNG port and jetties should include but not limited to the following: water conditions, land conditions, hydrological/meteorological conditions, geological conditions, seismic conditions, construction conditions, social support conditions, external pipeline layout, external supporting conditions, port services and management level, ship navigation conditions, compliance with planning, surrounding project relationships and impacts, project investment/schedule and other special important 
factors. Due to the complexity of site selection considerations, in order to facilitate the comparison of terminal sites, it is necessary to re-form a set of new indicators that are unrelated to each other to replace the original indicators.

\subsection{Establishment and optimization of assessment system based on principal component analysis}

Principal component method can apply in many aspects of multivariate statistical analysis. Its basic theory is to use ideology of decreasing dimension. We can build a new index system by using a certain of algorithm on the basis of multi-parameter and multi-sample data array. Though the quantity of indexes in new system decreased, they can reflex initial system's information more intensively. The new index parameters are called principle component. The key steps are as follows:

- $\quad$ Standardize $\mathrm{p}$ indexes value in $\mathrm{n}$ samples to obtain matrix $\mathrm{Z}$.

- $\quad$ Built correlation coefficient matrix $\mathrm{R}=\mathrm{ZZ} \mathrm{Z}^{\mathrm{T}} /(n-1)$, calculate $\mathrm{p}$ eigenvalues: $\lambda_{1} \geq \lambda_{2} \geq \lambda_{3} \cdots \geq \lambda_{\mathrm{p}} \geq 0$, then calculate their corresponding feature vector: $\mathrm{e}_{i}=\left(l_{1 i}, l_{2 i}, \ldots, l_{p i}\right)$.

- Calculate variance contribution rate: $\alpha_{\mathrm{i}}=\lambda_{i} / \sum \lambda_{i}$, then sort rate and make sure that when first $m$ indexes' accumulating rate more than 0.85 , we can regard these $\mathrm{m}$ principle components can reflect $\mathrm{p}$ indexes.

- $\quad$ Calculate n sample's principle component: $\mathrm{M}_{\mathrm{i}, \mathrm{j}}=\mathrm{Z}_{\mathrm{i}, \mathrm{j}} \times\left\lceil e_{1}, e_{2}, \ldots, e_{p}\right\rceil$.

The principal component extraction is carried out on the consideration factors, and then the classification and generalization are implemented to obtain the evaluation index system as shown in figure 1 .

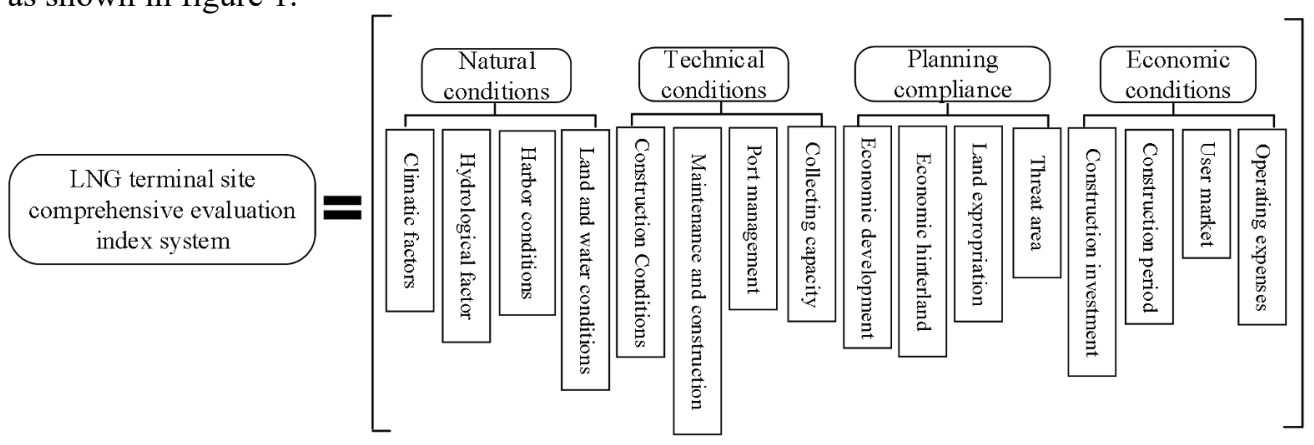

Fig. 1. Evaluation system.

\section{Principle of evaluation model based on cloud matter element theory}

Due to the characteristics of uncertainties in the evaluation process of LNG terminal site, and the evaluation level boundary information is ambiguous and random, the cloud matter element model is a combination of cloud model and matter-element theory which can process and solve this problem qualitatively and quantitatively.

\subsection{Cloud model}


The cloud model is a category of uncertainty artificial intelligence that converts between qualitative and quantitative. The uncertainty in nature is mainly random and ambiguous from the perspective of attributes. The cloud model explains the objective phenomenon law with double uncertainty through a unified mathematical expression [9].

\subsubsection{Concept of cloud model}

Cloud is the basic unit of the cloud model. The so-called "cloud" refers to a distribution on the domain $U$, which can be expressed in the form of joint probability $(x, u)$, that is, for $\forall \mathrm{x} \in U$, there is a random number with a tendency to be stable such that $\mu_{A}(x) \in[0,1]$. Where $\mathrm{U}$ is the quantitative domain composed of exact values; $\mathrm{A}$ is the qualitative concept on $\mathrm{U} ; \mu_{\mathrm{A}}(x)$ is the membership of $\mathrm{X}$ to $\mathrm{A}$.

\subsubsection{Digital characteristics of the cloud}

The cloud model can be represented by three data $\left(\mathrm{Ex}_{\mathrm{x}}, E_{n}, H_{e}\right)$ : Expectation $\mathrm{E}_{\mathrm{x}}$ : The expectation that the cloud droplets are spatially distributed in the universe is the point value that best represents the concept of the corresponding level boundary in the terminal.

Entropy $E_{n}$ : The degree of uncertainty depends on the degree of outliers and the degree of ambiguity. It also reflects the randomness of the sample data collected during the terminal evaluation process and the ambiguity of the sample data range. The smaller the entropy value, the smaller the double uncertainty of the sample data.

Super-entropy $\mathrm{He}_{\mathrm{e}}$ : used to measure the uncertainty of entropy, that is, the entropy of entropy, which is determined by the double uncertainty of entropy, which reflects the degree of dispersion of sample data.

The cloud membership function composed of the above three data of expectation, entropy and super-entropy can not only characterize the double uncertainty of the threshold value of the terminal evaluation level, but also soften the classification intervals.

\subsubsection{Normal cloud}

Cloud models have a variety of distribution patterns, such as trapezoidal clouds, normal clouds, and so on. Among them, normal clouds are widely used due to their unique mathematical properties. This paper analyses the normal cloud model according to its own needs [10].

\subsection{Matter-element analysis theory}

Matter-element theory refers to the use of primitives (including matter elements, events, and relationship elements) as logical cells, and qualitative and quantitative consideration of the qualitative and quantitative changes of things in a scalable way. The matter element is the basic cell in the theoretical analysis of matter element [11], which consists of the name of the thing $\mathrm{N}$, the feature of the thing $\mathrm{C}$, and the value $\mathrm{V}$ corresponding to the feature of the thing. The expression is $\mathrm{R}=(\mathrm{N}, \mathrm{c}, \mathrm{v})$. Among them, the feature element $\mathrm{M}(c, v)$ consisting of the feature name $\mathrm{C}$ and the corresponding magnitude $\mathrm{V}$ not only describes the 
characteristics of the thing, but also the relationship between the quality and quantity of the thing.

\subsection{Establishment of cloud matter-element model}

In the traditional matter-element analysis model, the threshold value or measured value of each indicator with certainty is represented by $\mathrm{V}$, but its double uncertainty is not considered [12]. In this paper, the cloud model has the unique advantage of dealing with the double uncertainty of things. The $\mathrm{v}$ value is replaced by the normal cloud $\left(\mathrm{E}_{\mathrm{x}}, E_{n}, H_{e}\right)$, and characterizing the ambiguity of the concept of the boundary level of the index through $\mathrm{E}_{\mathrm{n}}$, and portraying the randomness of the site assessment data to evaluate through $\mathrm{He}_{\mathrm{e}}$. The cloud matter model represents:

$$
R=\left[\begin{array}{ccc}
N & c_{1} & \left(E_{x 1}, E_{n 1}, H_{e 1}\right) \\
& c_{2} & \left(E_{x 2}, E_{n 2}, H_{e 2}\right) \\
\vdots & \vdots \\
& c_{n} & \left(E_{x n}, E_{n n}, H_{e n}\right)
\end{array}\right]
$$

\subsection{Establishment of level boundary cloud model}

In order to improve the credibility of the evaluation results, the Duffel method is used to divide the boundaries of the LNG terminal site selection criteria. The Duffel process is shown in figure 2. According to possible consequences of assessment, at first, we can divide the consequences into four class: best, good, fair, bad.

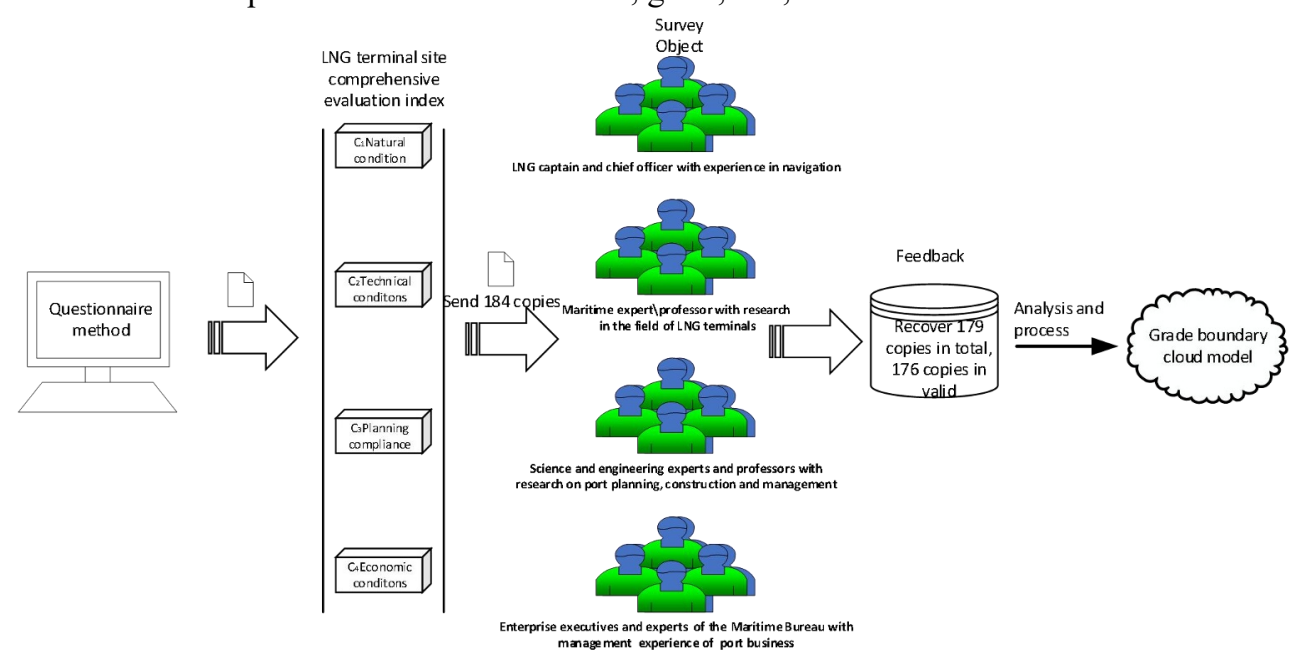

Fig. 2. Delphi method process.

The content of the questionnaire is the level of the second level index under the first level indicator given by each expert and the second level indicator judgment matrix established by the 9 scale method. The feature values of each judgment matrix are obtained, and the weight vector of the second indicator is obtained. The weight value of the secondlevel index is multiplied by the corresponding level of the second-level indicator. Finally, 
the corresponding level of each level-level indicator is summed to obtain the level of the first-level indicator. The results are shown in table 1.

Table 1. Grade limit of LNG terminal port site evaluation indices.

\begin{tabular}{|c|c|c|c|c|}
\hline \multirow{2}{*}{ Grade } & \multicolumn{4}{|c|}{ Evaluation index limit } \\
\cline { 2 - 5 } & $c_{1}$ & $c_{2}$ & $c_{3}$ & $c_{4}$ \\
\hline Best & $\geq 90$ & $\geq 85$ & $\geq 80$ & $\geq 88$ \\
\hline Good & $\geq 75$ & $\geq 75$ & $\geq 70$ & $\geq 76$ \\
\hline Fair & $\geq 60$ & $\geq 65$ & $\geq 60$ & $\geq 65$ \\
\hline Bad & $<60$ & $<65$ & $<60$ & $<65$ \\
\hline
\end{tabular}

Then, we can define the interval upper limit and lower limit as $\mathrm{C}_{\max }$ and $\mathrm{C}_{\min }$ before we calculate three eigenvalues. Using the following formula to calculate $E_{x}$ and $E_{n}$ :

$$
\begin{aligned}
& \mathrm{E}_{\mathrm{x}}=\left(C_{\min }+C_{\text {max }}\right) / 2 \\
& \mathrm{E}_{\mathrm{n}}=\left(C_{\max }-C_{\text {min }}\right) / 6 \\
& \mathrm{H}_{\mathrm{e}}=s
\end{aligned}
$$

$\mathrm{S}$ is a constant and is determined by the double uncertainty of the corresponding indicator, which can be determined according to the actual situation.

By equation 2, a standard normal cloud model for the terminal evaluation index is obtained [13], as shown in table 2 .

Table 2. Grade limit cloud model of LNG terminal port site integration evaluation indices.

\begin{tabular}{|l|c|c|c|c|}
\hline \multirow{2}{*}{ Grade } & \multicolumn{4}{|c|}{ Grade limit cloud model } \\
\cline { 2 - 5 } & $c_{1}$ & $c_{2}$ & $c_{3}$ & $c 4$ \\
\hline Best & $(95,1.6667,1.0000)$ & $(92.5,2.5000,1.0000)$ & $(90,3.3333,1.0000)$ & $\begin{array}{c}94,2.0000,1.000 \\
0)\end{array}$ \\
\hline Good & $\begin{array}{c}(82.5,2.5000,1.0000 \\
)\end{array}$ & $(80,1.6667,1.0000)$ & $(75,1.6667,1.0000)$ & $\begin{array}{c}(82,2.0000,1.000 \\
0)\end{array}$ \\
\hline Fair & $\begin{array}{c}(67.5,2.5000,1.0000 \\
)\end{array}$ & $(70,1.6667,1.0000)$ & $(65,1.6667,1.0000)$ & $\begin{array}{c}(70.5,1.8333,1.00 \\
00)\end{array}$ \\
\hline Bad & $(30,10.0000,2.0000)$ & $\begin{array}{c}(32.5,10.8333,2.0000 \\
)\end{array}$ & $\begin{array}{c}(30,10.0000,2.0000 \\
)\end{array}$ & $\begin{array}{c}(32.5,10.8333,2.0 \\
000)\end{array}$ \\
\hline
\end{tabular}

\subsection{Cloud matter-element correlation function}

Based on the correlation function calculation of the cloud matter element model, the correlation between the numerical value and the cloud matter element and the correlation degree between the cloud object element and the cloud matter element are determined. This article uses the former according to the characteristics of the assessment of the port. The calculation steps are as follows:

- Regard the value $\mathrm{X}$ as a cloud drop;

- Generating a random number $\mathrm{E}_{n}^{\prime}$ obeying a normal distribution, where the expected value is $E_{n}$ and the standard deviation is $\mathrm{H}_{\mathrm{e}}$;

- The degree of correlation between the value and the normal cloud matter element is obtained: 


$$
\mathrm{u}(x)=\exp \left(-\frac{\left(x-E_{x}\right)^{2}}{2\left(E_{n}^{\prime}\right)^{2}}\right)
$$

\section{Determination of the assessment rank based on the subjective and objective comprehensive weighting method}

\subsection{Determination of index weight coefficient}

In this paper, the subjective and objective comprehensive weighting method, which integrates the analytic hierarchy process [14] and the coefficient of variation method [15], is used to determine the weight coefficient of each index in the port evaluation. The expression is:

$$
\left[\begin{array}{c}
\omega_{1} \\
\omega_{2} \\
\vdots \\
\omega_{9}
\end{array}\right]=\left[\begin{array}{c|ccc}
c_{1} & A & S & R \\
c_{2} & A & S & R \\
& \vdots & S & R \\
c_{9} & A & S & R
\end{array}\right]
$$

In the formula, $\omega_{\mathrm{i}}$ represents the comprehensive weight value of indicator $\mathrm{c}_{i} ; \mathrm{A}$ represents the analytic hierarchy process of subjective weight assignment; $\mathrm{S}$ represents the coefficient of variation method for objective weight assignment method that can reduce human interference; $\mathrm{R}$ represents the synthesis between $\mathrm{A}$ and $\mathrm{S}$.

Let the subjective weight vector be $W_{s}$ and the objective weight vector be $W_{o}$.

Through the additive integration principle, the subjective weight value and the objective weight value are combined to obtain the final weight:

$$
\mathrm{W}=\mathrm{a} W_{s}+b W_{o}
$$

Assuming there are $\mathrm{n}$ indicators, then:

$$
\begin{aligned}
& \mathrm{a}=\frac{1}{n-1}\left(\sum_{i=1}^{n} i P_{i}-\frac{n+1}{n}\right) \\
& b=1-a
\end{aligned}
$$

where $\mathrm{P}_{\mathrm{i}}$ is the corresponding component from the small to large after the subjective weight vector is sorted in ascending order [16].

\subsection{Determination of the rank of the port assessment}

First, according to the correlation value between the to-be-evaluated index data calculated by the formula (3) and the normal cloud of each evaluation level standard, the comprehensive evaluation matrix $\mathrm{D}$ is composed of the correlation degree values.

Then, combined with the integrated weight coefficient $\mathrm{W}$, the comprehensive evaluation result vector is obtained from equation (7).

$$
\mathrm{B}=\mathrm{W} \cdot \mathrm{D}
$$

Finally, using the weighted average principle, the preliminary evaluation result $r$ is calculated from equation (8). 


$$
\mathrm{r}=\frac{\sum_{i=1}^{n} b_{i} f_{i}}{\sum_{i=1}^{n} b_{i}}
$$

In formula, $\mathrm{b}_{i}$ is the corresponding component of vector $\mathrm{B}, f_{i}$ is the score of the grade $\mathrm{i}$ to which the data to be evaluated belongs. According to 4 classes as mentioned before, we can rate them by using "4" "3" "2" "1" as responding 4 classes. The higher the rank, the higher the score.

In the process of calculating the correlation between the quantitative index $\mathrm{x}$ and the standard normal cloud using the correlation function [17], since $\mathrm{E}_{n}^{\prime}$ is randomly generated. In order to eliminate the random error, the final evaluation result, that is, the expected value $E_{r x}$ and the entropy $E_{m}$ are obtained according to the equations (9) and (10) after multiple calculations.

$$
\begin{aligned}
& E_{r x}=\frac{r_{1}(x)+r_{2}(x)+\cdots r_{h}(x)}{h} \\
& E_{r n}=\sqrt{\frac{1}{h} \sum_{i=1}^{h}\left[r_{i}(x)-E_{r x}\right]^{2}}
\end{aligned}
$$

In the formula: $\mathrm{h}$ is the number of times that $\mathrm{r}$ is calculated, and 100 calculations are performed in this paper; $r(x)$ is the evaluation score obtained by each operation.

The $E_{r x}$ value is a reflection of the level of the terminal port evaluation result. In order to measure the degree of dispersion of the evaluation result $\mathrm{r}(x)$, the entropy $\mathrm{E}_{\mathrm{m}}$ corresponding to the evaluation level is obtained, and the more concentrated the evaluation result, the smaller the value. In order to better measure and present the evaluation results, the confidence coefficient is defined as follows:

$$
\rho=\frac{E_{r n}}{E_{r x}}
$$

The magnitude of the $\rho$ value reflects the degree of dispersion of the evaluation results expressed by $\mathrm{E}_{\mathrm{rx}}$ and the degree of confidence as shown in table 3 .

Table 3. The meaning of $\rho$ value

\begin{tabular}{|c|c|c|c|}
\hline Reflecting results & $\rho$ & Dispersion of assessment results & Credible degree \\
\hline \multirow{2}{*}{$E_{r x}$} & Large & Large & Small \\
\cline { 2 - 4 } & Small & Small & Large \\
\hline
\end{tabular}

\section{Case study}

This paper takes two candidate terminal sites of an LNG project in China as an example for analysis and verification. The candidate ports are comprehensively evaluated by using the evaluation index system of figure 1. Through data statistics and analysis, the scores of the evaluation indicators of the two terminal ports are finally obtained, as shown in table 4 . 
Table 4. Index matrix of to-be-evaluated object.

\begin{tabular}{|l|c|c|}
\hline \multirow{2}{*}{ Evaluation indexes } & \multicolumn{2}{|c|}{ Indexes/score } \\
\cline { 2 - 3 } & $\mathrm{A}$ & $\mathrm{B}$ \\
\hline Natural conditions & 77.3470 & 76.9240 \\
\hline Technical conditions & 86.3610 & 73.6530 \\
\hline Planning compliance & 73.7420 & 84.8640 \\
\hline Economic conditions & 82.5750 & 77.4510 \\
\hline
\end{tabular}

The AHP is used to construct the evaluation index judgment matrix to obtain the eigenvalues, and the subjective weights of each indicator are obtained. Based on the indexes to be evaluated, the VCM is used to obtain the objective weights of the respective indicators. Finally, through the principle of additive integration, the subjective and objective comprehensive weights are calculated $(a=0.6968, b=0.3032)$. The results are shown in table 5 .

Table 5. The weight of each assessment indicator in site selection.

\begin{tabular}{|l|c|c|c|}
\hline Evaluation indexes & Subjective weight & Objective weight & Comprehensive weight \\
\hline Natural conditions & 0.1017 & 0.0149 & 0.0754 \\
\hline Technical conditions & 0.5485 & 0.4309 & 0.5129 \\
\hline Planning compliance & 0.2966 & 0.3805 & 0.3220 \\
\hline Economic conditions & 0.0532 & 0.1737 & 0.0897 \\
\hline
\end{tabular}

Table 6. Results comparison.

\begin{tabular}{|l|l|l|}
\hline Comparison method & A site & B site \\
\hline Literature [2] & Good & Good \\
\hline \multirow{2}{*}{ Method of this paper } & $\operatorname{Good}\left(E_{r x}=3.1223\right.$ & Good $\left(E_{r x}=3.1062\right.$ \\
& $\rho=0.0501)$ & $\rho=0.1519)$ \\
\hline Judgement result by expert & \multicolumn{2}{|c|}{ A site } \\
\hline
\end{tabular}

Using the correlation function, the correlation between the indexes to be evaluated and the normal cloud of each evaluation level is obtained, and a comprehensive evaluation matrix is constructed. Combined with the comprehensive weights of table 5, the comprehensive evaluation results are obtained, that is, the collection of evaluation results of each evaluation index. Using the formula (8), the preliminary evaluation results are obtained. Due to the randomness in the process of associating relevance, the randomness effect should be weakened. Through the formulas (9) to (11), the final evaluation results of the comparison port are determined, and the confidence coefficients are also obtained. The results obtained were compared with those in the literature [2] as well as the actual selection result. The comparison results are shown in table 6 .
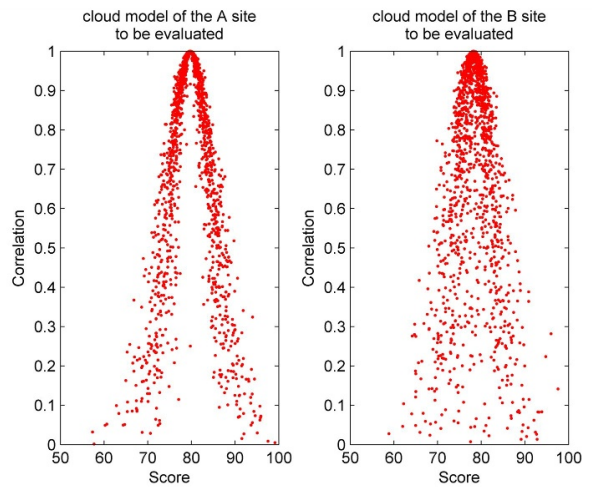

Fig. 3. The score cloud model distribution map of the site to be evaluated. 
By comparison of methods, the results of this paper are consistent with the results of the literature [2], and in line with the actual site selection. However, this paper analyses the location of the site from the perspective of qualitative and quantitative, not only gives reasonable location results, but also gives the degree of propensity and confidence information of each port. The experimental results show that the confidence of each evaluation result is in a small range, indicating that the evaluation results have high credibility. In the case where the A-level value is slightly higher than $\mathrm{B}$, and the confidence coefficient of A is significantly smaller than the B-confidence coefficient, this indicates that the evaluation result of $\mathrm{A}$ is more reliable. From a quantitative point of view, it is more certain that A should be preferred as the final site of construction.

In addition, from the distribution of the score cloud model of the two sites as shown in figure 3 [18], the A graph is more concentrated, indicating that the evaluation results are less dispersed and the corresponding confidence values are smaller. The distribution of the B cloud model is relatively scattered, indicating that the evaluation results are highly dispersed, due to the greater confidence value. This result fully complies with the change law described in table 3 , and fully reflects that the cloud matter element model has higher adaptability in the comparison and selection of the LNG terminal site.

\section{Conclusion}

The paper uses a combination of multiple models and multiple methods to couple the cloud model to the matter element, and the model adaptation space is improved. The association degree between the collected raw data and the established standard cloud matter model can be directly determined, which avoiding the loss of information due to data normalization processing. The method proposed in this paper can not only obtain objective and comprehensive evaluation results conveniently and effectively, but also reflect the degree of the same result level and the corresponding confidence information, which provides a new method for the comparison and selection of LNG terminal.

However, using the cloud matter-element model, we must first establish a standard indices system, and then measure the gap between the data to be evaluated and the standard, so as to determine the rank of the LNG terminal to be evaluated. This requires the scientific establishment of a standard indices system for LNG terminals as well as the classification. Different indices systems will result in different evaluation results. Therefore, in order to improve the universal applicability and feasibility of the evaluation method, the further research will focus on the analysis of the LNG terminal standard indices system and classification.

\section{Acknowledgment}

My heartfelt thanks appreciately express to all those who helped me during the writing of this thesis.Especially to Professor Dai,the second author of this paper,I am particularly indebted to him who gave me kind encouragement and countless support and instructions before the thesis finished.

\section{References}

1. Liu K, QIN J and Mi B Y 2012 Site selection of LNG terminal Marine Traffic Engineering 7 77-81

2. Hao Q L and Dai R 2013 Dalian LNG terminal port site selection based on fuzzy evaluation J. of Dalian Maritime Uni. (Nature Science) 39(3)103-06 
3. Jiang H Y and Wang W X 2004 Application of principal component analysis in synthetic appraisal for multi-objects decision-making J. of Wuhan Uni. of Tech. (Transportation Science \& Engineering) 3 467-70

4. Qi M F, Fu Z G, Jing Y et al. 2013 A comprehensive evaluation method of power plant units based on information entropy and principal component analysis Proc. of CSEE 2 $58-64+12$

5. ISBN 1856091295 Site Selection and Design for LNG Ports and Jetties

6. Ministry of Communications of PRC 2008 Design code of general layout for sea port (Beijing : China Communications Press)

7. Ministry of Communications of PRC 2001 Liquefied natural gas terminal design specification (Beijing: China Communication Press)

8. Taylor D W 2007 The role of consequence modeling in LNG facility siting J. Hazard. Mater. 142 776-85

9. Li D Y, Meng H J and Shi X M 1995 Following Cloud and Subordinate Cloud Generator J. of Computer Research and Development 06 15-20

10. Zhu Y L, Gong Z, Wu Z L, et al. 2010 The Application of Normal Cloud Model in Condition Assessment in Power Transformers J. of North China Electric Power Uni. (Natural Science Edition) 527-31

11. Hu Z B 2012 Study on transformer insulation condition assessment Based on cloud matter element theory Baoding:North China Electric Power Uni.

12. Li R Q and Su H Y 2012 A synthetic power quality evaluation model based on extension cloud theory Automation of Electric Power Systems 36(1) 66-70

13. Hong Q L, Zhang J T, Xie Q, et al. 2017 Transformer's condition assessment method based on combination of cloud matter Proc. of the 9th Asia-Pacific Power and Energy Engineering Conf. (Chengdu) pp 659-66

14. Deng X, Li J M, Zeng H J, et al. 2012 Analysis and application of the weight calculation method of analytic hierarchy process Mathematics Practice and Cognition 42(7) 94-100

15. Huang C H, Hu S P, Gao D Y, et al. 2013 Risk assessment of navigation safety in bridge water areas $J$. of Safety and Environment 13(6) 238-42

16. Zhuang W Y, Ling D, Zhao J, et al. 2006 Research on weights of agility appraisement indexes J. of Uni. of Electronic Science and Tech. of China 35(6) 985-88

17. Liu G H, Song C X and Liu H 2007 Software implementation of cloud generators Application Research of Computers 24(1) 46-48

18. Lv H J, Wang Y, Li D Y, et al. 2003 Application of reverse cloud in qualitative evaluation Chinese J. of Computers 26(8) 1009-14 